\title{
3-D Structure of Cellulose Microfibril in Wood Using Electron Tomography and Single Particle Reconstruction Methods
}

\author{
Mehedi Reza, Peter Engelhardt and Janne Ruokolainen \\ Department of Applied Physics, Aalto University, Espoo, Finland
}

Wood cells are consisted of different layers where they have cellulose, hemicelluloses and lignin. Cellulose in the form of microfibrils (MF) forms the skeleton of wood cell wall and is surrounded by lignin-hemicellulose matrix. Therefore it has been always a challenging job to observe the physical structure of cellulosic MF in wood cell wall. In our study we visualized spatial organization and physical structure of MF in 3-D using different methods. To see the spatial organization of $\mathrm{MF}$, we performed single-axis electron tomography. Ultrathin sections $(\sim 40 \mathrm{~nm}$ thick) were cut using an ultra-microtome (Leica EM FC7) equipped with diamond knife and was followed by staining with $\mathrm{KMnO}_{4}$. After staining gold particles were applied to the grids to act as fiducial markers. Tilt series was acquired using a Cryo-TEM (JEOL JEM-3200FSC) at 80k nominal magnification at an accelerating voltage of $200 \mathrm{kV}$. The specimen was tilted in the microscope from $-60^{\circ}$ to $+60^{\circ}$ with $3^{\circ}$ angular increment. Maximum entropy method was then used for data processing [1]. In the final volume, we found an average MF diameter of about $32 \AA$ (Figure 1) corresponding to a previous study [2]. The MFs are forming clusters where they reside so tightly and are surrounded by lignin-hemicellulose matrix.

In order to achieve better resolution and to observe individual MF with internal structure, we approached single particle reconstruction (SPR) methods. $\mathrm{KMnO}_{4}$ stained ultrathin sections ( $\sim 40$ $\mathrm{nm}$ thick) were used for Cryo-TEM at the accelerating voltages of $200 \mathrm{kV}$ and $300 \mathrm{kV}$. All the micrographs used for SPR were taken at 100k nominal magnification. Micrographs free from drift and astigmatism were selected for particle picking. At high magnification MFs appear like series of granular particles placed end to end [3]. From the images we have boxed $(64 \times 64$ pixel, 1 pixel $=$ $1.09 \AA$ ) up some hundred thousand repeating granular structures randomly from the sections that appear similar using a collecting method in sections that we call random extensive sampling (RES) using EMAN2's graphical interface workflow and/or projectmanager (Figure 2a) [4]. EMAN2 is an image processing suite for electron microscopy. As MFs are consisted of both crystalline and amorphous regions, we assume that the particles were picked from both regions. With EMAN2 we performed phase flipping of the picked particles. The orientations of particles are aligned and refined to produce a 3-D model of MF that appears about 20-32 $\AA$ in diameter and is inhomogeneous in thickness (Figure 2b). An initial model generated by EMAN1 with C1 symmetry was used for aligning and refining the particles. In the final 3-D volume we found a highly ordered region surrounded by a less ordered cortex region (Figure 2b). This result is supported by fine crystalline structures as shown in wood by X-ray diffraction [5]. Part of an interfibrilar bridge (IFB) is also visible in the final volume (Figure 2b) [6]. EMAN2's volume resolution program (e2eotest) indicated a resolution of $\sim 6 \AA$ by splitting data into even and odd halves and computing the Fourier shell correlation (FSC) between 3-D models (Figure 3).

Then we used the same particle set to another SPR method RELION, a Bayesian SPR method [7]. This method also confirmed the presence of a highly ordered region surrounded by a less ordered region (Figure 4) with a resolution of 3-4 $\AA$. Using a "gold-standard Fourier shell correlation" procedure RELION avoids "overfitting" resolution [7]. Our approach to SPR for sections appears to be a useful technique when it is impossible to isolate intact particles. 


\section{References}

[1] P Engelhardt, Encyclopedia of Analytical Chemistry. John Wiley \& Sons Ltd, Chichester (2000), pp. 4948-4984. Online: http://www.lce.hut.fi/ engelhar/EAC/ET-Cromosome-StructHR.pdf

[2] P Xu, LA Donaldson, ZR Gergely and LA Staehelin, Wood Science and Technology 41 (2007), p.101-116.

[3] AJ Hodge and AB Wardrop, Nature 165 (1950), p.272-275.

[4] EMAN: http://blake.bcm.edu/emanwiki/EMAN

[5] M Peura, M Müller, U Vainio, M-P Sarén, P Saranpää and R Serimaa, Trees 22 (2008), p.49-61

[6] LA Donaldson and AP Singh, Holzforschung 52 (1998), p.449-454

[7] SHW Scheres, J. Struct. Biology (2012), p.512-530.

[8] This work is supported by Multidisciplinary Institute of Digitalization and Energy (MIDE, http://mide.aalto.fi)

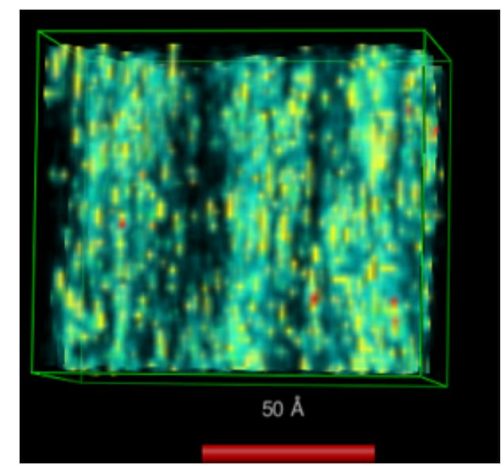

Figure 1. Cropped 3-D volume of three MF produced by electron tomography
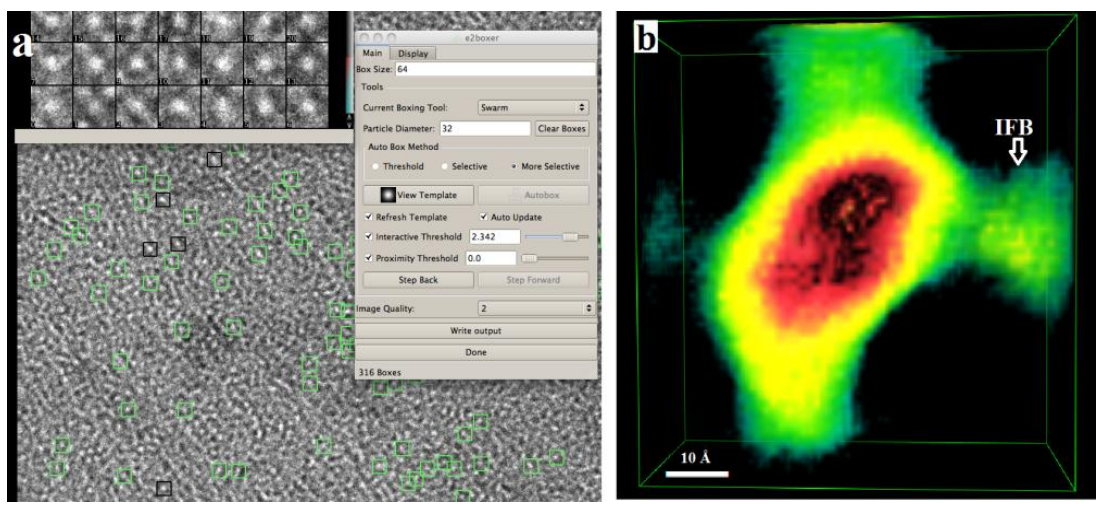

Figure 2. (a) Particle picking by EMAN2's "e2boxer", (b) SPR by EMAN2 with inside view

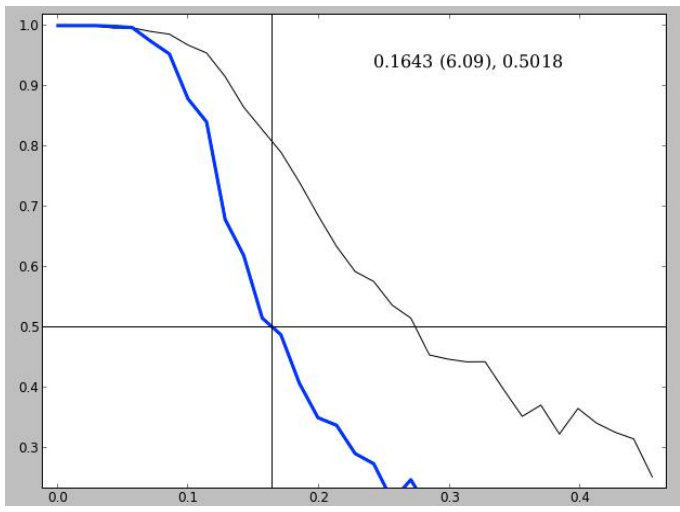

Figure 3. Resolution estimation by e2eotest tool using Fourier Shell Correlation

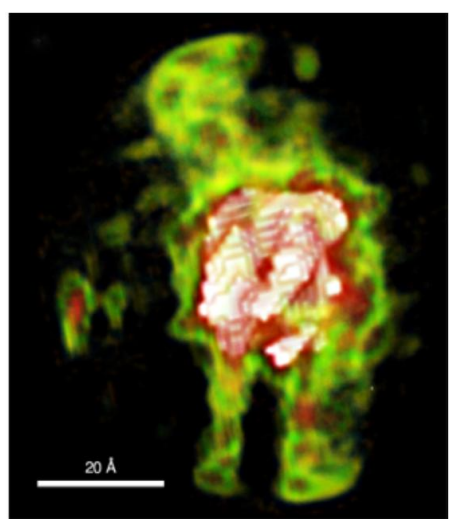

Figure 4. SPR by RELION 